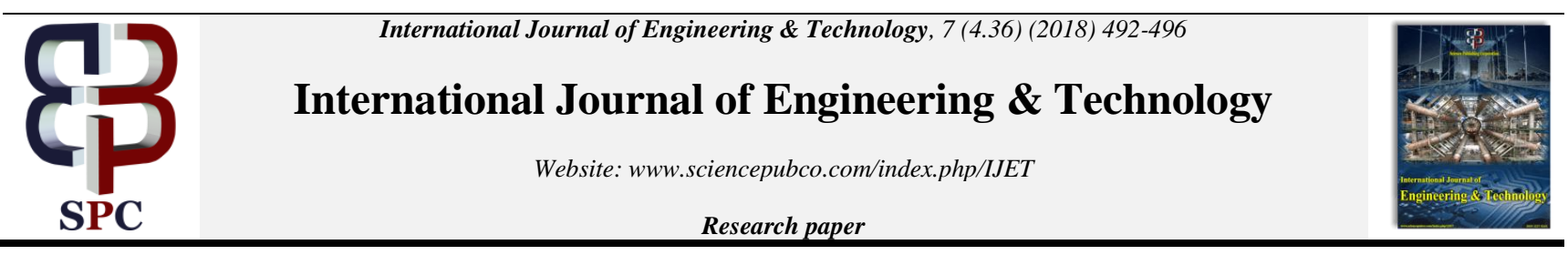

\title{
End-to-End Packet Delay Analysis and Modeling Concept in Multi-Hop Wireless Network
}

\author{
Arun Pratap Srivastava ${ }^{1 *}$, Shashank Awasthi ${ }^{2}$ \\ ${ }^{1}$ Department of Information Technology, ${ }^{2}$ Department of Computer Science \& Engineering \\ ${ }^{1,2}$ G. L. Bajaj Institute of Technology \& Management, Greater Noida, G. B. Nagar, India \\ *Corresponding author E-mail: apsvgi@gmail.com,
}

\begin{abstract}
Multi-hop remote innovation improve the a few properties (limit and scope) in a financially savvy way and it has been present in FourthGeneration (4G) norms. A multi-hop remote framework is can be clarified in better path like as multi-hop and time-opened yet the compelling limit idea is produced in view of single-jump and nonstop time correspondence frameworks. So two idea are utilized to portray the conduct of framework: the multi-jump successful limit idea for multi-bounce systems and the Mixed Continuous/DiscreteTime (MCDT) viable limit idea for time-opened systems coming about in this way there are two ways are include to approve these two idea on perfect remote interchanges, bundles navigate various remote system gadgets and parcels are transmitted to or gotten from a remote system gadget each Transmission Time Interval (TTI). So in the field of Multi-hop remote Network, the paper concept enhance the execution.
\end{abstract}

Keywords: End-to-End Packet Delay, Multi-Hop Wireless Network, Discrete-Time, and TDMA.

\section{Introduction}

Multi-hop wireless network is shown in figure 1, in which the communications between base stations and subscriber stations with the help of one or more relay stations.

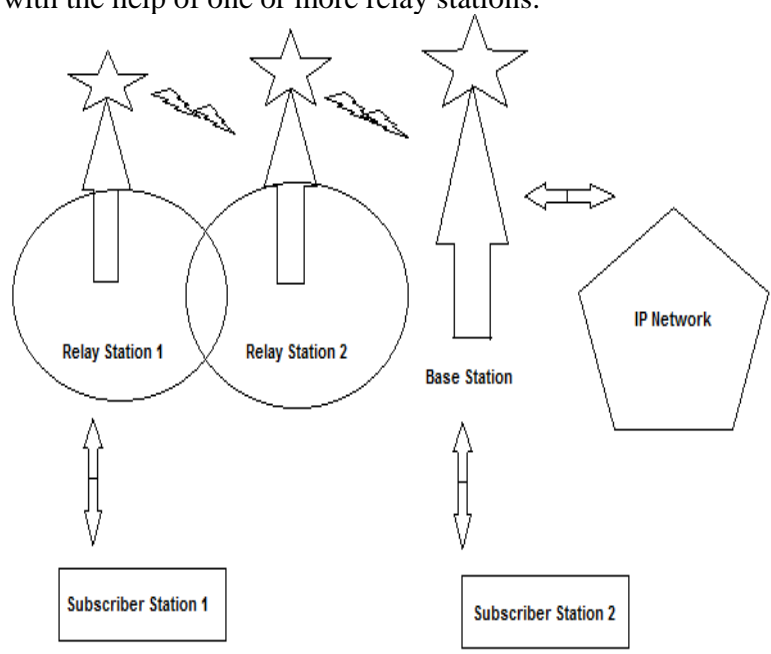

Figure 1 An example of multi-hop wireless networks.

As opposed to the arrangements of wired backhaul joins, the innovation gives a practical method to expanding system limit and scope. Therefore, such an innovation is bolstered by numerous standard remote models, for example, the Long Term EvolutionAdvanced (LTE-A) discharge 10 [1], the IEEE 802.16j [2] and the IEEE 802.11s [3]. The Quality of Service (QoS) is a principal segment of broadband remote correspondence systems since organize administrators or specialist co-ops focus to give an agreeable Internet administration to end clients so the conclusion to-end bundle delay is a QoS metric and it is essential for some uncommon deferral touchy applications, for example, Voice over Internet Protocol (VoIP), intuitive gaming and video conferencing. Clog and lines are non specific highlights of bundle exchanged systems. Parcel buffering now and again of system blockage is the principle component for supporting and smoothing bursty information movement, guaranteeing better throughput for these kinds of systems when contrasted with circuit-exchanged systems [4]. This is the principle properties was the fundamental explanation behind the ubiquity of bundle exchanged systems as a rule and Internet Protocol (IP) organizes specifically to connect PCs. The disadvantage of utilizing cradles is that they likewise present unusual and variable defers when bundles navigate crosswise over systems. Before the late 1990s, information organizes chiefly exchanged "flexible" (non-time-delicate) movement in customary applications, for example, File Transfer Protocol (FTP), email and web perusing. The Service of value measurements reflect negative impacts of blockage in bundle exchanged systems and are normally identified with three system execution measurements $[4,5]$ :

1. Deferral: Packets are conveyed to goal hubs with delays, which may differ from parcel to bundle (one measure is jitter).

2. Throughput (or conveyed information rate): Different movement composes require diverse information rates.

3. Misfortune: Packets neglect to achieve their goals. Misfortunes can happen in the physical layer, interface layer or potentially organize layer [6]. 


\section{Multi-hop Wireless Networks}

The primary recognizing normal for a multi-hop remote system is its capacity of self-association and multi-hop interchanges [7]; these present progressive architecture [8]. Multi-hop wireless networks are classified in two types:

1. Mobile Multi-hop Relay (MMR) networks and

2. Wireless Mesh Networks (WMNs).

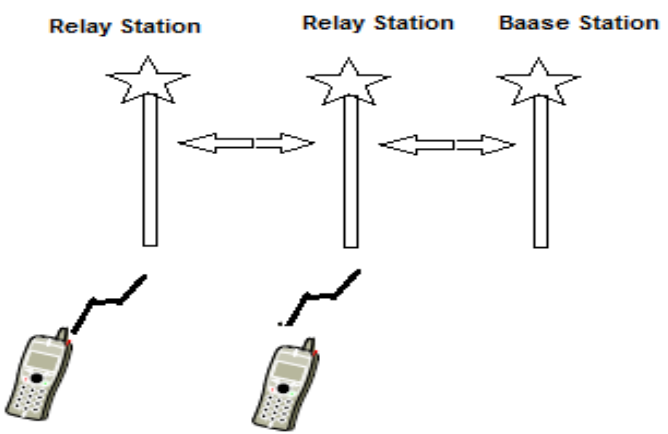

Fig (2) Mobile Multi-hop Relay (MMR) networks

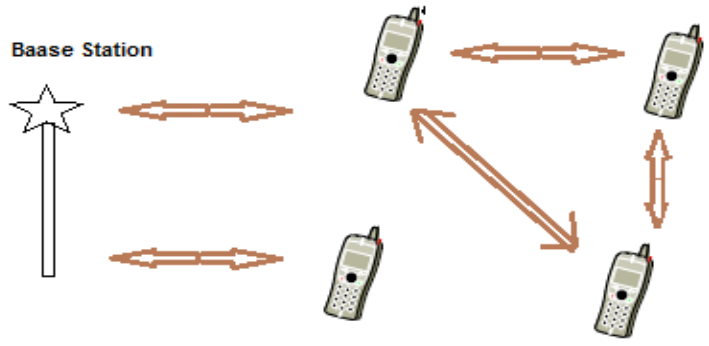

Fig (3) Wireless Mesh Networks (WMN)

In Mobile Multi-hop Relay organizes, the system engineering comprises of base stations, relay stations and subscriber stations. A relay station isn't straightforwardly associated with wired framework and has the base usefulness important to help multihop correspondences. The essential perspective is that subscriber station to subscriber station correspondence ways need to incorporate a base station or a hand-off station. (Fig 2)

In Wireless Mesh Networks, movement can be steered through other subscriber stations and can likewise happen specifically between them. Hubs are involved work switches and work customers and subsequently directing procedure is controlled by base stations or hand-off stations as well as by subscriber stations. Every hub can forward bundles for the benefit of different hubs that may not be inside direct remote transmission scope of their goal. A framework that has an immediate association with backhaul benefits outside the work arrange is named a work base station. The various frameworks are called work supporter stations. Business organization situations of the remote work innovation were considered in [8]. (Fig 3)

As Compared to centralise networks the multi-hop wireless technology brings many benefits-

- Routing activity Flexibility: The capacity of picking the privilege directing way would significantly spare data transmission assets by limiting the movement.

- Enhancement of Capacity: The telephone edge zone and a dead zone district (caused by the radio blockage) generally have cut down Signal-to-Interference-and-Noise extent (SINR) values than various locales inside the telephone. Hence, conveying transfer hubs or work switches in such region defeats this issue and enhance the framework execution. Encourage limit pick up: The multi-hop design presents the space decent variety so it empowers helpful numerous information various yield [9] innovation. Other propelled methods can be consolidated into multi-hop structures to additionally upgrade limit, for example, subjective radio [10] and versatile pre-coding [11].

- Scalability: Nodes go about as repeaters to transmit information from adjacent hubs to peers that are too far away to reach [12], bringing about a system that can traverse substantial separations, particularly finished unpleasant or troublesome landscape.

- Reliability: Resilience to unit disappointment with the arrangement of reinforcement ways.

- Cost-sparing: Deploying hand-off stations or work switches is more monetarily powerful than sending base stations, making the multi-hop remote innovation a perfect answer for organize administrators (particularly for those destitute Internet Service Providers (ISPs) and transporters) to take off hearty and solid remote broadband administration access with satisfactory in advance venture [12]

- Energy proficiency: The multi-hop design can altogether lessen the vitality utilization in circumstances where clients need to make a considerable measure of high information rate associations. At the point when there is no movement, hand-off stations or work switches may additionally spare vitality by changing to the lowvitality remain by mode [13]

- Potential for high ghastly efficiencies: In physical work acknowledgment, directional reception apparatuses and brilliant receiving wires could be utilized to enhance the transmission in multi-hop remote systems $[14,15]$.

Nonetheless, the multi-hop remote innovation additionally presents a few difficulties:

- QoS ensures: The conclusion to-end defer is related with channel conditions and number of hop, both of which are hard to be ensured.

- Security in WMNs: Until now, there has been no brought together trusted expert to appropriate an open key in WMNs because of its circulated framework design [16]. Without a persuading security arrangement, the remote work innovation will be unable to succeed in light of the fact that clients may do not have the impetus to buy in to questionable administrations.

- Timing synchronization in time division various access (TDMA) Systems [17]: TDMA frameworks have a high necessity for the time synchronization, yet in a disseminated multi- hop arrange, exact synchronization inside the worldwide system is hard to accomplish.

- Support for steering usefulness and appropriateness flagging: The extraordinary attributes of multi-hop remote systems propose that they request a particular answer for the help for directing usefulness and respectability flagging, which is as yet an open inquiry [18].

Based on the Open Systems Interconnection (OSI) 7-layer display, there are three sorts of the multi-bounce remote innovation [19]:

1. The layer-1 transfer (the hand-off station is known as a supporter or repeater): As appeared in Fig. 4, it is an increase forward kind of hand-off innovation. Radio repeat signals got on the downlink from the base station are increased and transmitted to the versatile station. In a comparable way, radio recurrence signals got on the uplink from the mobile station are increased and transmitted to the base station. 

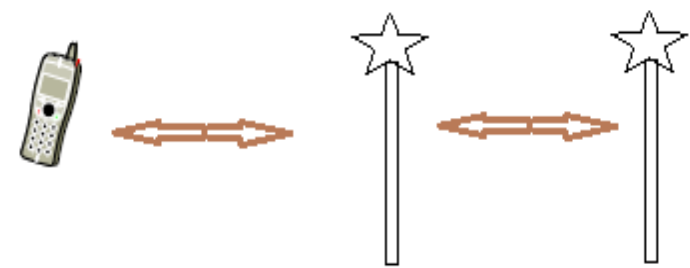

Layer-1 Relay

Station / Repeater

\begin{tabular}{|c|}
\hline Power \\
Amplification
\end{tabular}

Fig 4- Amplify-Forward type

2. The layer-2 transfer: As appeared in Fig. 5, it is an interpret forward kind of hand-off innovation. radio frequency signals got on the downlink from the base station are demodulated and decoded and a short time later encoded and changed again before being sent on to the versatile station.

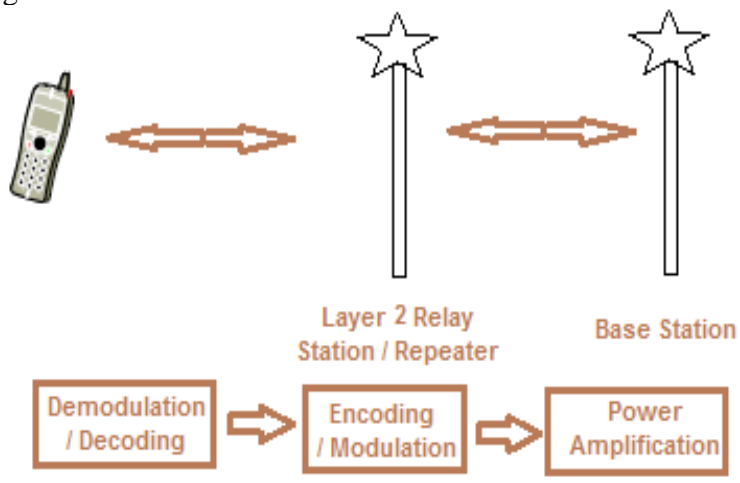

Fig 5- Decode-Forward type

3. The layer-3 transfer: As appeared in Fig. 6, it additionally performs demodulation and deciphering of radio recurrence signals got on the downlink from the build station, anyway then goes in light of to perform getting ready, (for instance, figuring and customer data association/division/reassembly) to retransmit customer data on a radio interface in conclusion performs encoding/modification and transmission to the versatile station.

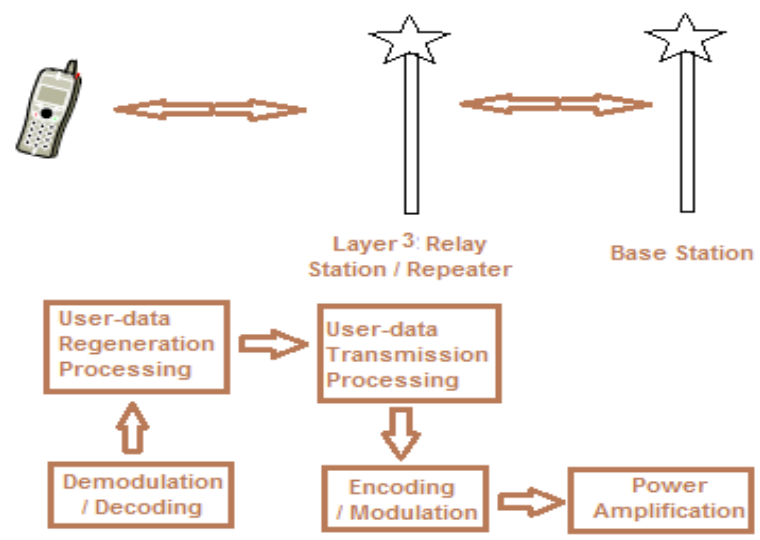

Fig 6- The layer-3 relay

The layer-1 relay is the least difficult sort of hand-off so it makes for ease execution and short preparing delays. In any case, the layer-1 hand-off opens up between cell obstruction and commotion together with wanted flag segments in this way decaying the got SINR and diminishing the throughputimprovement pick up [19].

The layer- 2 and layer- 3 relay play out the demodulation and translating preparing at hand-off stations, which beats the disadvantage in layer-1 hand-off of breaking down got SINR caused by intensification of between cell obstruction and commotion. Along these lines, these two sorts can accomplish a superior throughput-upgrade.

Fundamentally there are three standard of Multi-hop Wireless Technology are presented; Include Long Term EvolutionAdvanced (LTE-A), the IEEE 802.16j and the IEEE 802.11s.

I-The LTE- the LTE is institutionalized by the third Generation Partnership Project (3GPP) Release 10 [20]. The LTE-A will be a product update for the Long Term Evolution (LTE) systems; and this standard requires top download rates more than 1gigabit, which completely bolsters the $4 \mathrm{G}$ necessities in view of the International Telecommunication Union - Radio-correspondence Sector (ITU-R). There are a few key upgrades and increments of new highlights to the LTE, among which is the MMR innovation. ii-The IEEE 802.16 is a progression of remote broadband benchmarks with a few arrivals of these measures to help new advances. The popularized mark name of the IEEE 802.16 standard is Worldwide Interoperability for Microwave Access (WiMAX) [21]. The WiMAX innovation offers high information rates over a generally expansive scope territory and its current advancements from both scholarly world and industry are reviewed in [22].

iii-The IEEE 802.11s is inside the standard group of the IEEE 802.11 (some of the time known as Wi-Fi). The essential thoughts of the IEEE $802.11 \mathrm{~s}$ is that 1 ) it utilizes 802.11 -based physicallayer gadgets and connection layer conventions for giving the usefulness of an Extended Service Set (ESS) work system, and 2) it incorporates a few changes for work organizing, characterizing how remote gadgets can interconnect to make a specially appointed network.[23]

\section{End-to-End Packet Delay concept}

End-to-end bundle delay is the time taken for a parcel to be transmitted over a system from source to goal; its formal definition is-End-to-end bundle defer Denote by EEn the time moment of the nth bundle entry (the last piece or the most huge piece of the bundle has been gotten) at a system and indicate by $\mathrm{Tn}$ the time moment of the nth bundle flight (the last piece or the most critical piece of the parcel has been transmitted) from the system. The conclusion to-end postpone of the nth parcel DEn (or the deferral of the bundle touches base at moment EEn, DE(EEn)) can be scientifically ascertained from

$\mathrm{DEn}=\mathrm{DE}(\mathrm{EEn})=\mathrm{Tn}-\mathrm{EEn}$

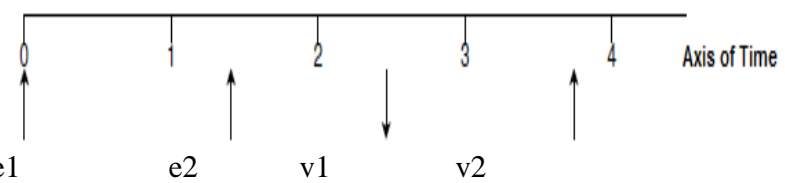

Fig 7 example of packet arrivals and departures

A case of parcel landings and takeoffs is appeared in Fig. 7. It important that

- The documentation of DE(EEn) is usually utilized as a part of liquid movement models.

- $\mathrm{D} \infty$ or $\mathrm{D}(\infty)$ indicates the conclusion to-end postpone when a framework achieves its unfaltering state;

- Packet is a question idea. It can allude to an IP bundle, a video edge et cetera.

In most correspondence frameworks, end-to-end parcel delay are arbitrary factors since bundle entries and takeoffs are stochastic procedures.

A conclusion to-end postpone process $\{\mathrm{DEn}, \mathrm{n} \geq 1\}$ is a requested succession of bundle end-to end delays from the first parcel; and it is a stochastic procedure. Consider an acknowledgment $\{\mathrm{d} 1, \mathrm{~d} 2$, $\mathrm{d} 3, \cdot, \mathrm{dN}\}$ of a conclusion to-end postpone process with $\mathrm{N}$ samples.[24]. 


\section{Modelling of End-to-End Packet Delay}

The Modeling of End-to-End Packet Delay is portray by the diverse models and hypotheses

I-Model for Data Flow

ii-Delay in the Model of Fluid and Packetised

iii-Theory of Network Calculus

iv-Model of Effective Capacity

$\mathrm{v}$-Lindley Equation

A switch or router can be simply modelled as a queuing system like Fig. 8

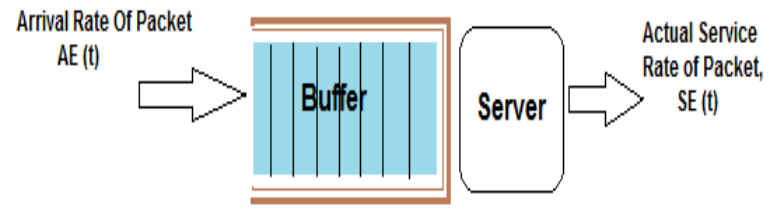

Fig 8- Queuing Mode

The system can be for the most part portrayed utilizing Kendall's documentation $[25,26]$ (it is the standard documentation used to depict and group a lining hub) as $\mathrm{A} / \mathrm{S} / \mathrm{c} / \mathrm{K} / \mathrm{D}$, in which

- A indicates a between entry appropriation;

- $\mathrm{S}$ indicates an administration time conveyance;

- $\mathrm{c}$ indicates the quantity of servers;

- $\mathrm{K}$ indicates the cradle estimate (the most extreme bits that a cushion can hold);

- D indicates a queue discipline.

The investigation of the behavior of a queue models is called queuing theory.The primary paper on lining hypothesis was distributed in 1909 by Danish mathematician Agner Krarup Erlang [27]. From that point forward, the queuing theory has been consistently considered and has broad outcomes [26]. In addition, the system math hypothesis is another branch of lining hypothesis spearheaded by R.L.Cruz [28, 29]. The theory gives a queuing system for investigating execution ensures in PC organizes and incorporates the compelling limit demonstrate, which describe end-to-end defer appropriations in remote systems.

\section{i- Model for Data Flow-}

Fundamentally there are two kinds of time domain, i.e., continuous time and discrete time and the model of Data stream can be grouped into two models:

1. Continuous time model: $\mathrm{t} \in \mathrm{R}+\mathrm{o}=[0, \infty)$;

2. discrete-time model: the model is time-opened and is watched just at space limits.

$\mathrm{t} \in \mathrm{N}=\{0,1,2,3, \ldots\}$.

In genuine frameworks, there is dependably a base granularity (bit, word, cell or bundle), along these lines discrete time could simply be expected [30]. Nonetheless, it is regularly computationally less complex to consider continuous time.

A persistent time display and discrete-time demonstrate are portray by the diverse procedure i.e. landing process, aggregate entry process, benefit process, combined administration process, real piece benefit process and total real administration process.

\section{ii- Delay in the Model of Fluid and Packetised-}

There are two kind of models are consider which can be exist in continuous-time model and discrete-time model

1. Fluid traffic model:: bundle sizes are imperceptibly little or parcels are arrived a tiny bit at a time;

2. Packetised traffic model: bundles have non-insignificant sizes.

The packetised traffic model is a more sensible model than the fluid traffic model since parcel sizes are typically non-unimportant in down to earth circumstances. This model further enables us to think about queuing delays of packets. In media transmission and information organizes, the conclusion to-end packet delay DEn for the most part comprises of four components [31]:

1. Transmission delay DE(t)n : the Transmission delay is define a time required for a packet to be transmitted at the transmitter

2. Radio propagation Delay DE(r)n : The Radio propagation Delay is the time which is requires for a parcel to achieve its beneficiary 3. Signal processing delay $\mathrm{DE}(\mathrm{s}) \mathrm{n}$ : The Signal processing delay is the time which is required for a parcel to be decoded at the collector,

4. Queuing delay $\operatorname{DE}(q) n$ : The Queuing delay is the time which is required for a bundle to hold up in cushions or the time between the parcel entry moment and the moment when the main piece or the slightest critical piece was sent.

Their scientific connection can be essentially communicated as $\mathrm{DEn}=\mathrm{DE}(\mathrm{t}) \mathrm{n}+\mathrm{DE}(\mathrm{r}) \mathrm{n}+\mathrm{DE}(\mathrm{s}) \mathrm{n}+\mathrm{DE}(\mathrm{q}) \mathrm{n}$

Expecting that the radio transmission postponement and flag handling delay are sufficiently little to disregard, so condition might be composed as DEn $=\mathrm{DE}(\mathrm{t}) \mathrm{n}+\mathrm{DE}(\mathrm{q}) \mathrm{n}$

\section{iii- Theory of Network Calculus-}

In wired systems, the theory of network calculus is an examination device to define the Quality of Service (QOS), for example, the Asynchronous Transfer Mode (ATM) systems and the Internet [28]. The movement portrayal in the network calculus theory requires the information measurement (i.e., bits as an element of time) created by a source adjust to an upper bound, which is known as traffic envelope $\Gamma E(\mathrm{t})$ [32]. So also, the service characterization $¥ \mathrm{E}(\mathrm{t})$ [33] for ensured benefit in the network calculus theory is an assurance of a base administration rate. Capacities $\Gamma E(t)$ and $¥ \mathrm{E}(\mathrm{t})$ are indicated as far as certain traffic and service parameters, individually. For example- A leaky container is a gadget that shapes the entry rate $\mathrm{AE}^{\prime}(\mathrm{t})$. The container of this gadget is at first unfilled and it can hold up to $\sigma \mathrm{E}(\mathrm{s})$ bits. The container likewise has an opening and holes at a rate of $\lambda \mathrm{E}(\mathrm{s}) \mathrm{s}$ bit every second $(\lambda \mathrm{E}(\mathrm{s}) \mathrm{s}$ is named as the feasible rate) when it isn't vacant.

\section{iv- Model of Effective Capacity-}

The remote channels have low dependability and time-differing limits, this create serious QoS infringement. Accordingly, it is smarter to describe remote channels from a measurable view point. The effective capacity model is an examination instrument for demonstrating the conclusion to-end postpone in a stochastic way and it was proposed by Wu and Negi in 2003 [34]. After the approach of the effective capacity model, various endeavors were made to ascertain the compelling limit work in different correspondence frameworks and channels [35, 36], utilize the successful limit capacity to improved frameworks [37, 38], create applications in light of the model and estimator [39, 40], and change the model for more sensible correspondence situations [39].

\section{v Lindley Equation for Delay-}

The main Lindley condition for the holding up times of the clients was proposed in 1952 by D. V. Lindley [41]. Since the condition is connected to any $\mathrm{G} / \mathrm{G} / 1 / \infty / \mathrm{FIFO}$ queuing model, it is regularly viewed as a general frame for

- delay processes

- Continuous-time or discrete-time models

- packetised traffic models only

Let TEn, CEn and DE(q)n mean the arbitrary factors of the nth between entry time and the service time of the $n$ packet and the queuing delay value of the nth parcel, individually. 
For a G/G/1/o/FIFO queuing model, The $\mathrm{DE}(\mathrm{q}) \mathrm{n}$ will be$\mathrm{DE}(\mathrm{q}) \mathrm{n}=\max \{0, \mathrm{DE}(\mathrm{q}) \mathrm{n}-1+\mathrm{CEn}-1-\mathrm{TEn}-1\}$

This condition define the relation or connection between the queuing delay of the $n$th and the service time of the $(n-1)$ st packet and the $(n-1)$ st between entry time. On the off chance that TEn or CEn is an arbitrary variable, $\mathrm{DE}(\mathrm{q}) \mathrm{n}$ is an irregular variable.

\section{Conclusion}

The idea of End-to-End Packet Delay examination and Modeling Concept in Multi-Hop Wireless Network is tied with parcel exchanging correspondence frameworks and QoS measurements incorporate bundle delay, through and packet loss ratio. At the point when delay-touchy activity is conveyed in system, instruments for giving end-to-end delay ensures are required. When all is said in done, this paper reviewed the ideas that identifies with multi-hop wireless network systems, parcel deferral and end-to-end demonstrating of bundle delay.

The multi-hope wireless technology is a characteristic mix of centralised networks and ad-hoc networks. This innovation answers numerous intense issues experienced in wireless communication system, similar to no man's land issue and the sending in the unforgiving territory. Its focal points, challenges were altogether researched, together with its three sorts of executions.

\section{References}

[1]. Yu Chen, Jia Chen and Yang Yang, "Multi-hop Delay Performance in Wireless Mesh Networks", MONET 2008, Vol. 13 (published before the PhD started)

[2]. Yu Chen, Yang Yang and Izzat Darwazeh, "A Cross-Layer Analytical Model of End-to-end Delay Performance for Wireless multi-hop Environments", IEEE GLOBECOM 2010, Miami, USA, December 2010

[3]. $\mathrm{Yu}$ Chen and Izzat Darwazeh, "End-to-end Delay Performance Analysis in IEEE 802.16j Mobile multi-hop Relay (MMR) Networks", IEEE ICT 2011, Ayia Napa, Cyprus, May 2011.

[4] N. Olifer and V. Olifer, Computer Networks: Principles, Technologies and Proto-cols For Network Design. Wiley India Pvt. Limited, 2006.

[5] A. Tanenbaum, Computer networks, ser. Computer Science. Prentice Hall PTR, 2003.

[6] J. F. Kurose and K. Ross, Computer Networking: A Top-Down Approach Fea-turing the Internet, 2nd ed. Boston, MA, USA: Addison-Wesley Longman Publishing Co., Inc., 2002.

[7] P. Mach and R. Be`st"ak, "Wireless mesh and relay networks," in Research in Telecommunication Technology 2006, 2006

[8] R. Bruno, M. Conti, and E. Gregori, "Mesh networks: commodity multihop adhoc networks," Communications Magazine, IEEE, vol. 43, no. 3, pp. 123 - 131, march 2005.

[9] A. Sadek, W. Su, and K. Liu, "Multinode cooperative communications in wireless networks," Signal Processing, IEEE Transactions on, vol. 55, no. 1, pp. 341-355,2007.

[10] J. Mitola and J. Maguire, G.Q., "Cognitive radio: making software radios more personal," Personal Communications, IEEE, vol. 6, no. 4, pp. 13-18, 1999.

[11] C. Masouros and T. Ratnarajah, "Utilization of primary-secondary crossinterference via adaptive precoding in cognitive relay assisted mimo wireless systems," in Communications (ICC), 2011 IEEE International Conference on, 2011, pp. 1-5.

[12] Y. Zhang, J. Luo, and H. Hu, Wireless Mesh Networking: Architectures, Protocols and Standards, 1st ed. Auerbach Publications, Dec. 2006.

[13] I. Haratcherev and A. Conte, "Practical energy-saving in $3 g$ femtocells," in Communications Workshops (ICC), 2013 IEEE International Conference on, 2013, pp. 602-606.

[14] P. Piggin, B. Lewis, and P. Whitehead, Mesh networks in Fixed Broadband Wireless Access. Radiant Networks PLC, Essex UK, 2003.143

[15] S. Bellofiore, J. Foutz, R. Govindaradjula, I. Bahceci, C. A. Balanis, A. S. Spanias, J. M. Capone, and T. M. Duman, "Smart antenna system analysis, integration and performance for mobile ad hoc networks (manets), 2005.

[16] I. F. Akyildiz, X. Wang, and W. Wang, "Wireless mesh networks: a survey," Computer Networks (Amsterdam, Netherlands: 1999), vol. 47, no. 4, pp. 445-487, Mar. 2005.

[17] (2014) Network Time Synchronization Bibliography. http://www.eecis.udel.edu/ mills/biblio.html.

[18] . Sonia, . Raouf, . Youssef, and . Brent, "Routing protocols in wireless mesh networks: challenges and design considerations," 2006.

[19] M. Iwamura, H. Takahashi, and S. Nagata, "Relay technology in lteadvanced," NTT DoCoMo Technical Journal, vol. 12, no. 2, pp. 29-36, 2010.

[20] E. Seidel, "White paper on lte advance," Nomor Research, Tech. Rep., July 2008.

[21] WiMAX.com. (2010) Wimax reaches "mass-market," devices to triple in 2010. Online, Available: http://www.wimax.com/wimax/wimax-reaches-qmassmarketqdevices-to-triple-in-2010.

[22] M. Ma, Current Technology Developments of WiMax Systems. Springer, 2009

[23] B. G. Lee and S. Choi, Broadband Wireless Access and Local Networks: Mobile WiMAX and WiFi, 1st ed. Artech House Publishers, May 2008. 144.

[24] M. Woodward, Communication and computer networks: modelling with discrete-time queues. Pentech, 1993.

[25] D. G. Kendall, "Stochastic Processes Occurring in the Theory of Queues and their Analysis by the Method of the Imbedded Markov Chain," The Annals of Mathematical Statistics, vol. 24, no. 3, pp. 338-354, 1953.

[26] D. Gross, J. F. Shortle, J. M. Thompson, and C. M. Harris, Fundamentals of Queueing Theory, 4th ed. New York, NY, USA: Wiley-Interscience, 2008.

[27] A. K. Erlang, "The Theory of Probabilities and Telephone Conversations," Nyt Tidsskrift for Matematik, vol. 20, no. B, pp. 33-39, 1909.

[28] R. Cruz, "A calculus for network delay. i. network elements in isolation," Information Theory, IEEE Transactions on, vol. 37, no. 1, pp. $114-131$, Jan 1991.

[29] R. L. Cruz, "A calculus for network delay. ii. network analysis," vol. 37, no. 1,pp. 132-141, 1991 .

[30] J. L. Boudec and P. Thiran, Network Calculus: A Theory of Deterministic Queuing Systems for the Internet, 1st ed. Springer, Aug. 2001

[31] D. Bertsekas and R. Gallager, Data Networks. Prentice-Hall, 1987.

[32] T. Athanasiadis, Y. Avrithis, and S. Kollias, "The atm forum. traffic management specification version 4.0 (1996)," Tech. Rep., 1996.145

[33] R. Gu'erin and V. Peris, Quality-of-service in Packet Networks: Basic Mechanisms and Directions. IBM Watson Research Center, 1998.

[34] D. Wu and R. Negi, "Effective capacity: a wireless link model for support of quality of service," Wireless Communications, IEEE Transactions on, vol. 2, no. 4, pp. 630 - 643, July 2003.

[35] K. Angrishi and U. Killat, "Analysis of a real-time network using statistical network calculus with effective bandwidth and effective capacity," pp. 1-15, 2008, measuring, Modelling and Evaluation of Computer and Communication Systems (MMB), 2008 14th GI/ITG Conference.

[36] S. Akin and M. C. Gursoy, "Effective capacity analysis of cognitive radio channels for quality of service provisioning," vol. 9, no. 11, pp. 3354-3364, 2010.

[37] L. Musavian and S. Aissa, "Quality-of-service based power allocation in spectrumsharing channels," in Proc. IEEE Global Telecommunications Conf. IEEE GLOBECOM 2008, 2008, pp. 15.

[38] — , "Adaptive modulation in spectrum-sharing systems with delay constraints," in Proc. IEEE Int. Conf. Communications ICC '09, 2009, pp. 1-5.

[39] D. Wu and R. Negi, "Downlink scheduling in a cellular network for quality-ofservice assurance," vol. 53, no. 5, pp. 1547-1557, 2004. 147

[40] _ "Effective capacity channel model for frequency-selective fading channels," in Proc. Second Int Quality of Service in Heterogeneous Wired/Wireless Networks Conf, 2005.

[41] Al-Fatlawi, A.A.Y. " Anti-proliferative and pro-apoptotic activity of carvacrol on human cancer cells",(2018) International Journal of Pharmaceutical Research, 10 (2), pp. 235-240. 